\title{
SPIRE (ALEXIS), WEIDENFELD (KATIA), L'IMPUNITÉ FISCALE. QUAND L'ÉTAT BRADE SA SOUVERAINETÉ, PARIS, LA DÉCOUVERTE, «L'HORIZON DES POSSIBLES », 2015, 176 PAGES.
}

\section{Jonathan Miaz}

De Boeck Supérieur | « Politix »

$2016 / 4 n^{\circ} 116$ | pages 226 à 229

ISSN 0295-2319

ISBN 9782807390409

Article disponible en ligne à l'adresse :

https://www.cairn.info/revue-politix-2016-4-page-226.htm

Distribution électronique Cairn.info pour De Boeck Supérieur.

(C) De Boeck Supérieur. Tous droits réservés pour tous pays.

La reproduction ou représentation de cet article, notamment par photocopie, n'est autorisée que dans les limites des conditions générales d'utilisation du site ou, le cas échéant, des conditions générales de la licence souscrite par votre établissement. Toute autre reproduction ou représentation, en tout ou partie, sous quelque forme et de quelque manière que ce soit, est interdite sauf accord préalable et écrit de l'éditeur, en dehors des cas prévus par la législation en vigueur en France. Il est précisé que son stockage dans une base de données est également interdit. 


\title{
SPIRE (Alexis), WeIDENFELD (Katia), L'impunité fiscale. Quand l'État brade sa souveraineté, Paris, La Découverte, «L'horizon des pos- sibles ", 2015, 176 pages.
}

\author{
Par Jonathan MIAZ \\ Doctorant en science politique, Université de Lausanne et Université de Strasbourg
}

Avec la crise de 2008 et la succession de scandales financiers qui s'est ensuivie, l'évasion et la fraude fiscales ont de plus en plus souvent été au centre de l'attention médiatique et politique. L'ouvrage d'Alexis Spire et Katia Weidenfeld sur l'impunité fiscale tombe donc à point nommé. Les auteurs partent du constat que bien que fortement réprouvée dans les discours, la fraude fiscale, qui soustrait chaque année au budget de l'État français entre 60 et 80 milliards d'euros (p. 6), fait l'objet d'une grande tolérance de la part des pouvoirs publics qui s'abstiennent notamment de recourir à l'arme pénale contre les fraudeurs. Ces derniers bénéficient donc d'une large impunité : les grandes entreprises et les contribuables les plus fortunés s'en sortent souvent avec des amendes ou avec des sursis, alors que les organisateurs de la fraude ne sont jamais mis en cause. Ce sont les ressorts de cette impunité que l'ouvrage entend mettre à jour.

Cet ouvrage constitue ce faisant une contribution importante à la compréhension de la délinquance des élites (pour une synthèse récente sur la question, cf. le livre de Pierre Lascoumes et Carla Nagels, Sociologie des élites délinquantes. De la criminalité en col blanc à la corruption politique, Armand Colin, 2014) et du traitement administratif et judiciaire des illégalismes fiscaux. Il s'inscrit dans la continuité des travaux historiques de Nicolas Delalande sur le consentement et les résistances à l'impôt (Les batailles de l'impôt. Consentement et résistances de 1789 à nos jours, Le Seuil, 2014) et sur le développement de la fiscalité (Delalande (N.), Spire (A.), Histoire sociale de l'impôt, La Découverte, 2010). Il prolonge également les publications de ses auteurs qui s'intéressent depuis plusieurs années au recours à la justice administrative (Contamin (J.-G.), Saada (E.), Spire (A.), Weidenfeld (K.), Le recours à la justice administrative. Pratiques des usagers et usages des institutions, La Documentation française, 2008) et aux inégalités entre faibles et puissants face à l'impôt (Spire (A.), Faibles et puissants face à l'impôt, Raisons d'Agir, 2012).

Le livre s'appuie sur un riche matériau empirique, à la fois historique et sociologique, permettant d'appréhender tout au long des maillons de la chaîne de la répression des illégalismes fiscaux, les logiques et les causes qui participent à la tolérance de la fraude et à l'impunité des plus puissants. Il repose d'abord sur une enquête qualitative menée entre 2012 et 2015 parmi tous les acteurs impliqués dans la répression de la fraude fiscale: inspecteurs des impôts, responsables départementaux, fonctionnaires de l'administration centrale, magistrats du siège et du parquet, enquêteurs, etc. Un des points forts du livre réside dans la combinaison de ces données qualitatives avec une analyse quantitative mobilisant des statistiques publiques et impliquant la constitution d'une base de données anonyme à partir de 570 affaires de fraude fiscale jugées en 2011 permettant de saisir les propriétés sociales des contribuables mis en cause.

La démonstration est organisée en six chapitres. Les deux auteurs affirment d'emblée que l'impunité fiscale est «directement inscrite dans la procédure qui organise un système de filtres successifs vers les poursuites pénales» (p. 8). La "machine à trier» les infractions fiscales s'apparente ainsi à " un véritable entonnoir » : à partir 
des 52000 contrôles fiscaux approfondis en 2013, 16300 fraudeurs sont démasqués, 1200 plaintes sont proposées et 1000 sont effectivement poursuivis devant le tribunal correctionnel. Le propos du livre est alors de "comprendre les mécanismes qui concourent en France à la persistance de l'impunité fiscale » et de "montrer que le traitement de cette délinquance profite particulièrement aux puissants et aboutit à un renoncement de l'État à sa propre souveraineté »(p.13-14). Pour les auteurs, si l'impunité fiscale n'est pas «le résultat d'une volonté délibérée, de la part des inspecteurs des impôts et des juges, de protéger les élites ", " ils y sont incités par un ensemble de contraintes institutionnelles et symboliques qui les dépassent largement » (p. 15).

En premier lieu, les auteurs montrent comment cette tolérance à la fraude fiscale est en fait ancrée dans l'histoire des lois et des pratiques. L'écart entre les discours qui réprouvent fermement les fraudeurs fiscaux et la rareté des sanctions effectivement prononcées à leur encontre est très ancien. Parmi les résistances à l'impôt, seules les oppositions frontales sont condamnées de manière intransigeante, alors que l'évitement discret de l'impôt, en jouant sur les règles, bénéficie d'une certaine indulgence. Ainsi, si les sanctions prévues par la loi peuvent apparaître conséquentes (amendes, peines de prison), " en pratique, les poursuites correctionnelles n'atteignent que les contribuables qui se refusent absolument à tout dialogue avec l'administration » (p. 42). Tout au long de la procédure, il existe des moyens de recours, de négociation et de transaction qui permettent aux auteurs de fraude fiscale d'éviter de se retrouver devant le juge pénal. Dès lors, « ce qui est visé, ce n'est pas le fait d'avoir éludé l'impôt, mais d'avoir manifesté, par cette résistance au fisc, son refus d'adhérer aux principes et aux valeurs de l'État » (ibid.).

L'étude du profil des contribuables mis en cause devant les tribunaux pour fraude fiscale indique qu'il existe un régime de sanctions à deux vitesses. D'un côté, ceux qui savent jouer de la législation et masquer l'intentionnalité de leurs transgressions ou reconnaître leurs fraudes bénéficient d'une régulation négociée. D’un autre côté, ceux dont les manquements sont évidents subissent un régime de sanction unilatérale pouvant aller jusqu'à la poursuite pénale. Ainsi, «les puissants échappent aux juges » (p. 44): les grandes entreprises, les contribuables des classes dominantes, de même que les intermédiaires et organisateurs de la fraude, qu'ils soient banquiers, avocats ou conseillers en patrimoine, s'en sortent soit par des amendes, soit en négociant leur impunité. Au contraire, "les faibles sont poursuivis» (p. 58) : les petits entrepreneurs de certains secteurs (bâtiment et construction, gardiennage et nettoyage), les étrangers, les gérants de paille ou les simples contribuables qui refusent de dialoguer avec le fisc se retrouvent davantage devant les juges.

Plusieurs raisons permettent d'expliquer la rareté des poursuites à l'égard des grandes entreprises et des contribuables les plus fortunés. Leurs dossiers se caractérisent d'abord par leur technicité et leur complexité. Les professionnels du droit et de la comptabilité n'y sont pas étrangers, en ce qu'ils participent à euphémiser leurs pratiques de contournement de l'impôt et à les présenter comme de simples optimisations. La technicité des montages juridiques constitue ainsi un obstacle de taille pour prouver l'intentionnalité du délit. Ensuite, la nécessité d'obtenir un recouvrement rapide place le rapport entre l'administration et les représentants des grandes entreprises ou les grandes fortunes sur le mode de la négociation, privilégiée au détriment de sanctions pénales. Ainsi, si les contrôles fiscaux couvrent un large spectre de contribuables, l'action pénale touche principalement des gérants d'entreprise de faible envergure et des petits entrepreneurs mal entourés. Cette " sélection paradoxale » s'explique par les filtres successifs tout au long de la procédure pénale qui agissent comme un véritable entonnoir. 
La justice elle-même fait preuve de passivité à l'égard des fraudeurs fiscaux, tant en raison de la place de ce délit dans les représentations des magistrats, de l'insuffisance des moyens dont ils disposent, mais aussi d'effets structurels. Il faut d'abord mentionner le fait que le contentieux fiscal est très marginal pour la justice pénale : quantitativement $(0,15 \%$ des délits jugés), du fait que les magistrats sont peu formés au droit fiscal et parce que l'intervention de l'institution judiciaire est toujours tardive dans ce domaine, plusieurs années après les faits. Ainsi, en matière de fraude fiscale, les procureurs jouent très peu le rôle de pilotage qu'ils ont habituellement dans l'institution judiciaire. Ce n'est qu'exceptionnellement que le procureur de la République confie des enquêtes pour fraude fiscale à un juge d'instruction, de sorte que les dossiers sont rarement plus étoffés après être passés par son bureau. Ainsi, « dans la majorité des cas, les magistrats du parquet restent dépendants de la mise en forme opérée par l'administration fiscale. Intervenant plusieurs années après les faits, l'institution judiciaire renonce le plus souvent à trouver les véritables organisateurs de la fraude fiscale et à dévoiler son ampleur ou ses motivations » (p. 102).

C'est aussi au niveau des peines prononcées que se situe la démobilisation de l'institution judicaire observée au niveau des procureurs. De manière générale, le traitement judiciaire est particulièrement clément par rapport à la criminalité en col blanc en général et plus encore par rapport aux autres domaines du droit pénal. Moins d'un fraudeur sur dix est condamné à une peine de prison ferme. Ici aussi, les intermédiaires du droit, des avocats fiscalistes, jouent un rôle important en mettant en exergue la complexité technique de la loi pour établir un doute quant à l'intentionnalité du délit. La place que ce dernier occupe dans les représentations des juges et dans l'inconscient collectif doit aussi être mentionnée : la fraude fiscale constitue en effet un délit sans victime et apparaît moins grave que les violences aux personnes ou les atteintes aux biens. Durant le procès, l'avocat mandaté pour représenter l'administration des finances ne met pas suffisamment l'emphase sur le tort causé, sur les motivations du prévenu ou sur sa dangerosité sociale. Il se consacre surtout à démontrer l'existence du délit et endosse dès lors peu la posture de victime. Au final, les sanctions prononcées par les juges semblent moins avoir pour fonction de punir que de faire rentrer l'argent dans les caisses de l'État.

Au final, ayant constaté que la machine à pénaliser la fraude fiscale «tourne, depuis plusieurs décennies, dans le vide»(p.149), les auteurs mobilisent des expériences étrangères (Grande-Bretagne, États-Unis) pour « remettre en cause ce qui paraît inéluctable» et « explorer l'horizon des possibles» (p. 133), à partir duquel les auteurs formulent des pistes afin d'améliorer la lutte contre l'impunité fiscale. Ils expliquent notamment que l'on pourrait mieux sélectionner les personnes poursuivies en portant les élites devant les juridictions pénales et en faisant primer dans certains cas l'objectif de punition des infractions sur la volonté de faire rentrer l'argent fraudé. Les promoteurs de fraude fiscale, les intermédiaires, pourraient aussi être mis en cause. L'institution judiciaire doit disposer de davantage de moyens afin de découvrir des faits inaccessibles aux agents du fisc. Avec cela, l'extension des compétences de la "police fiscale » et la suppression de la Commission des infractions fiscales - filtre supplémentaire en France ayant de puissants effets de sélection - sont des préalables pour réduire les délais et renforcer la transparence de la procédure pénale. Les auteurs proposent aussi de restituer un sens aux sanctions pénales, ce qui passe par exemple par le développement d'un récit autour de la fraude fiscale, visant à lui donner les traits d'une véritable délinquance. L'enjeu est d'incarner le délit et de rendre compréhensible le cadre juridique dans lequel il s'inscrit. On peut encore imaginer l'ouverture de l'éventail des peines au-delà de l'alternative entre la prison ferme, la prison avec sursis 
et l'amende : travaux d'intérêt général, interdictions d'exercer une profession, publicité autour des condamnations, etc. Pour les auteurs, les sanctions doivent être en adéquation avec l'objectif des poursuites pénales. Il ne s'agit pas seulement d'intimider, mais aussi de donner à la peine une fonction "socio-pédagogique ", ainsi que d'exprimer et de renforcer les normes et les valeurs sur lesquelles se fonde la société (p. 150).

La lecture de ce livre ouvre plusieurs pistes de recherche. Pour n'en citer que quelques-unes, l'approfondissement de la compréhension du rôle des intermédiaires du droit et de la comptabilité : comment jouent-ils avec les règles, souvent transnationales? Quel est leur rapport à la légalité, à l'impôt et à son contournement? Les mêmes questionnements peuvent d'ailleurs s'appliquer aux élites concernées : du fait de leur position sociale, comment ces dernières participent-elles à créer les conditions juridiques et sociales de leur propre impunité ? Le transnationalisme qui caractérise les délits de fraude fiscale mériterait aussi qu'on s'y attarde pour saisir comment les fraudeurs et leurs conseillers en jouent, comment s'y confrontent les autorités administratives et judiciaires ou, plus généralement, comment les États interagissent entre eux sur ce point.

L'impunité fiscale est donc un ouvrage particulièrement stimulant. S'il revêt un intérêt académique indéniable, il constitue aussi un apport au débat citoyen en formulant des propositions concrètes à la faveur de la comparaison avec d'autres pays et en défendant une "véritable pénalisation de la fraude fiscale» (p. 164). Cette dimension plus engagée constitue à notre sens un des intérêts de ce livre, en ce qu'il concilie, avec succès, une analyse historique et sociologique fine avec une réflexion citoyenne et la formulation de propositions pratiques. 